\title{
A Bioinformatics Tool for the Prediction of DNA N6-Methyladenine Modifications Based on Feature Fusion and Optimization Protocol
}

OPEN ACCESS

Edited by:

Yungang $X u$

University of Texas Health Science Center at Houston, United States

Reviewed by:

Dariusz Mrozek,

Silesian University of

Technology, Poland

Renzhi Cao,

Pacific Lutheran University,

United States

*Correspondence:

Guobao Xiao

gbx@mju.edu.cn

Leyi Wei

weileyi@tju.edu.cn

tThese authors have contributed equally to this work

¥Present address:

Ran Su,

School of Computer Software at

Tianjin University, Tianjin, China

Specialty section:

This article was submitted to

Bioinformatics and Computational

Biology,

a section of the journal

Frontiers in Bioengineering and Biotechnology

Received: 17 February 2020 Accepted: 29 April 2020

Published: 04 June 2020

Citation:

Cai J, Wang D, Chen R, Niu Y, Ye X,

Su R, Xiao G and Wei L (2020) A Bioinformatics Tool for the Prediction of DNA N6-Methyladenine Modifications Based on Feature Fusion and Optimization Protocol. Front. Bioeng. Biotechnol. 8:502. doi: 10.3389/fbioe.2020.00502

\author{
Jianhua Cai ${ }^{1,2 t}$, Donghua Wang ${ }^{3 \dagger}$, Riqing Chen ${ }^{4}$, Yuzhen Niu ${ }^{1}$, Xiucai Ye ${ }^{5}$, Ran Su ${ }^{6 \ddagger}$, \\ Guobao Xiao ${ }^{1 *}$ and Leyi Wei ${ }^{1,7 *}$
}

\begin{abstract}
${ }^{1}$ Fujian Provincial Key Laboratory of Information Processing and Intelligent Control, College of Computer and Control Engineering, Minjiang University, Fuzhou, China, ${ }^{2}$ College of Mathematics and Computer Science, Fuzhou University, Fuzhou, China, ${ }^{3}$ Department of General Surgery, Heilongjiang Province Land Reclamation Headquarters General Hospital, Harbin, China, ${ }^{4}$ College of Computer and Information Sciences, Fujian Agriculture and Forestry University, Fuzhou, China, ${ }^{5}$ Department of Computer Science, University of Tsukuba, Tsukuba, Japan, ${ }^{6}$ College of Intelligence and Computing, Tianjin University, Tianjin, China, ${ }^{7}$ School of Software, Shandong University, Jinan, China
\end{abstract}

DNA $N^{6}$-methyladenine $(6 \mathrm{~mA})$ is closely involved with various biological processes. Identifying the distributions of $6 \mathrm{~mA}$ modifications in genome-scale is of great significance to in-depth understand the functions. In recent years, various experimental and computational methods have been proposed for this purpose. Unfortunately, existing methods cannot provide accurate and fast $6 \mathrm{~mA}$ prediction. In this study, we present $6 m A P r e d-F O$, a bioinformatics tool that enables researchers to make predictions based on sequences only. To sufficiently capture the characteristics of $6 \mathrm{~mA}$ sites, we integrate the sequence-order information with nucleotide positional specificity information for feature encoding, and further improve the feature representation capacity by analysis of variance-based feature optimization protocol. The experimental results show that using this feature protocol, we can significantly improve the predictive performance. Via further feature analysis, we found that the sequence-order information and positional specificity information are complementary to each other, contributing to the performance improvement. On the other hand, the improvement is also due to the use of the feature optimization protocol, which is capable of effectively capturing the most informative features from the original feature space. Moreover, benchmarking comparison results demonstrate that our 6mAPred-FO outperforms several existing predictors. Finally, we establish a web-server that implements the proposed method for convenience of researchers' use, which is currently available at http://server.malab.cn/6mAPred-FO.

Keywords: DNA N6-methyladenine site, machine learning, feature representation, sequence-based predictor, feature fusion

\section{KEYPOINTS}

- In this study, we present 6mAPred-FO, a powerful bioinformatics tool for the prediction of $6 \mathrm{~mA}$ sites.

- In $6 \mathrm{mAPred}-\mathrm{FO}$, we integrate the sequence-order information with nucleotide positional specificity information for feature encoding, and further improve the feature representation capacity by feature optimization. 
- Comparative results showed that the proposed 6mAPred-FO significantly outperforms several existing predictors.

- We have established a webserver implementing the proposed $6 \mathrm{mAPred-FO}$. It is publicly accessible at http://server.malab. $\mathrm{cn} / 6 \mathrm{mAPred}-\mathrm{FO}$.

\section{INTRODUCTION}

$\mathrm{N}^{6}$-methyladenine $(6 \mathrm{~mA})$, as a dynamic DNA epigenetic modification, has been extensively discovered in the following three species: bacteria, archaea and eukaryotes (O'Brown and Greer, 2016). The newly studies have indicated that $6 \mathrm{~mA}$ modification participates in a wide spectrum of important biological processes. In prokaryotes, for example, $6 \mathrm{~mA}$ has been found to be closely correlated with a series of DNA activities, such as replication (Campbell and Kleckner, 1990; Li et al., 2019), repair (Pukkila et al., 1983), transcription (Robbins-Manke et al., 2005), and cellular defense (Luria and Human, 1952; Linn and Arber, 1968; Meselson and Yuan, 1968). In addition, some studies have demonstrated that $6 \mathrm{~mA}$ can act as an epigenetic mark in Phytophthora genomes and there may be a relationship between patterns of $6 \mathrm{~mA}$ methylation and adaptive evolution in these important plant pathogens (Chen H. et al., 2018). Besides, recent study demonstrated that DNA $6 \mathrm{~mA}$ modification plays a significant role in cell fate transition of mammalian cells as well (Liang et al., 2016; Liao et al., 2016). Therefore, it is very indispensable to determine the distribution of $6 \mathrm{~mA}$ modification sites in genome-scale to systematically interpret its biological functions.

To solve this problem, experimental efforts have been proposed, such as ultra-high performance liquid chromatography coupled with mass spectrometry (UHPLC$\mathrm{ms} / \mathrm{ms}$ ) (Greer et al., 2015), capillary electrophoresis and laser-induced fluorescence (CE-LIF) (Krais et al., 2010), methylated DNA immunoprecipitation sequencing (MeDIPseq) (Pomraning et al., 2009), and single-molecule real-time sequencing (SMRT-seq) (Flusberg et al., 2010). Notably, using mass spectrometry together with SMRT-seq, Zhou et al. obtained the first $6 \mathrm{~mA}$ profile in rice genome (Zhou et al., 2018). Currently, there is a publicly available database namely "MethSMRT" that integrates multiple $6 \mathrm{~mA}$ datasets derived from SMRT-seq (Ye et al., 2017). Although considerable progress has been made, the use of the high-throughput sequencing techniques is very limited as it is laborious and expensive.

Recently, as the rapid increase of the experimentally validated $6 \mathrm{~mA}$ sites, more research efforts have been focused on the development of data-driven computational methods, especially machine learning based prediction methods. For instance, Chen et al., proposed the first machine learning based $6 \mathrm{~mA}$ site predictor, named "i6mA-Pred," to predict $6 \mathrm{~mA}$ sites in rice genome (Chen et al., 2019). The i6mA-Pred used nucleotide chemical properties and nucleotide frequency as features to formulate DNA sequences (Chen et al., 2017) and utilized support vector machine (SVM) to train the predictive model (Chen et al., 2019). The i6mA-Pred model achieved $83.13 \%$ in terms of the overall accuracy for identifying $6 \mathrm{~mA}$ sites (Chen et al., 2019). More recently, researchers have proposed to use deep learning to identify $6 \mathrm{~mA}$ sites, like iDNA6mA (5-step rule)
(Tahir et al., 2019). This model can automatically extract features from DNA sequences by convolution neural network (CNN). Although these models have been proven to be effective and efficient in identifying DNA $6 \mathrm{~mA}$ sites, the accuracy was not high enough to perform the genome-wide prediction.

In this study, we propose a new bioinformatics predictor, namely "6mAPred-FO." In this predictor, we aim to capture the discriminative characteristics of $6 \mathrm{~mA}$ sites by differentview information integration and optimization. Based on the sequential features we extracted, we trained an SVM-based prediction model. Benchmarking comparative results have shown that under the 10 -fold cross-validation, our model improves the exiting performance to $87.44 \%$ in the overall accuracy. Via further experimental analysis, we found that our performance improvement contributes mainly to our feature integration and optimization strategy. In particular, the nucleotide positional specificity information is complementary to sequence-order information to effectively distinguish $6 \mathrm{~mA}$ sites from non-6mA sites. We anticipate this tool can be useful to discover new $6 \mathrm{~mA}$ sites in other species, at least complementary to the high-throughput techniques.

\section{MATERIALS AND METHODS}

\section{Benchmark Dataset}

A high-quality benchmark dataset is essential for building an effective and unbiased supervised learning model. In this study, we used the same stringent benchmark dataset, which is originally proposed in Chen's study (Chen et al., 2019). In the dataset, the positive samples (sequences with $6 \mathrm{~mA}$ sites) were obtained from NCBI Gene Expression Omnibus and the singlemolecule real-time sequencing (Zhou et al., 2018). Afterwards, they separated out the sites with a modification score of $<30$ according to the Methylome Analysis Technical Note, and used the CD-HIT (Fu et al., 2012) software to eliminate sequences with the similarity of more than $60 \%$ (Chen et al., 2019). The negative samples (sequences without $6 \mathrm{~mA}$ sites) were obtained from sub-sequences containing GAGG motifs in coding sequences (CDSs) of the rice genome (Zhou et al., 2018). Ultimately, 880 $6 \mathrm{~mA}$ sequences (positive samples) and 880 non- $6 \mathrm{~mA}$ sequences (negative samples) were retained in the dataset.

\section{Framework of the Proposed 6mAPred-FO}

Figure 1 illustrates the overall framework of the $6 \mathrm{mAPred}$ FO method for DNA $6 \mathrm{~mA}$ site prediction. The predictive procedure can be concluded as two phases: model training and prediction. In the training phase, the training samples are encoded and integrated by two feature representation algorithms: NPS (Nucleotide Positional Specificity) and PseDNC (Pseudo Dinucleotide Composition). Afterwards, the features are optimized to obtain the best feature subset for the training set. The resulting feature vectors are then fed into the SVM algorithm to train predictive model. In prediction phase, given the query sequences that are not characterized, we followed the similar procedure to encode the sequences, and used the trained model to predict whether the query sequences are $6 \mathrm{~mA}$ sites or not. 


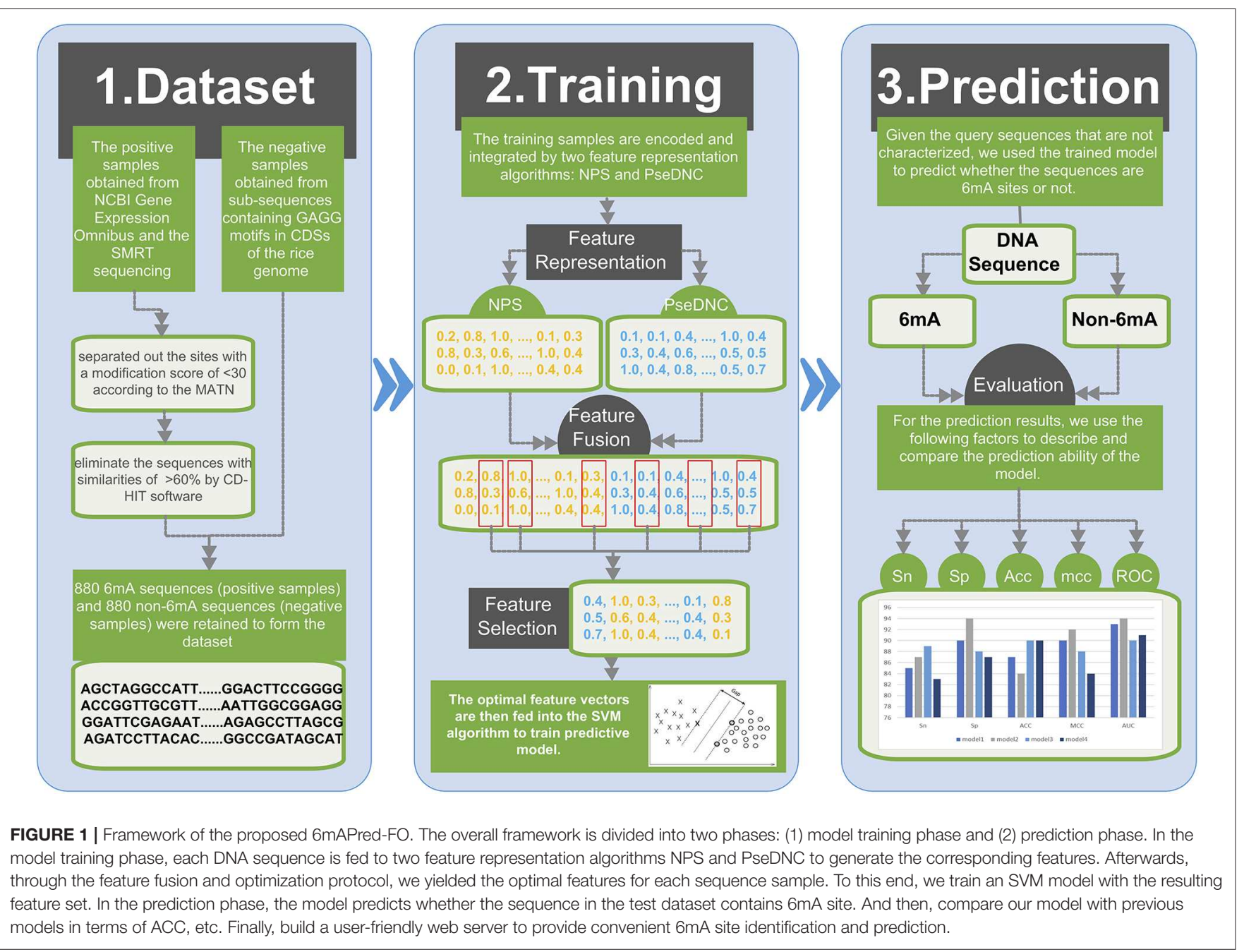

\section{Feature Representation Algorithms}

To convert DNA sequences into feature vectors that machine learning methods can handle, two feature representation algorithms, Nucleotide Positional Specificity (NPS) and Pseudo Dinucleotide Composition (PseDNC), are introduced for feature representation. Here is a brief introduction to the two algorithms.

\section{Nucleotide Positional Specificity (NPS)}

In this algorithm, two feature representation descriptors are used to encode the sequences.

The first feature is the positional binary encoding of flanking nucleotide sequence. We adopt the traditional method of flanking window to represent the $6 \mathrm{~mA}$ site. On the premise that the minimum length 41 can perform well, if the $6 \mathrm{~mA}$ site is located at both ends of the sequence, we fill the end of the sequence with the gap character "N." Therefore, in the orthogonal binary coding scheme, we transform nucleotide sequences into numeric vectors by the following rules: the codes of "A (adenine)," "T (thymine)," "C (cytosine)," "G (guanine)" and "N" are “ $(0,0,0,1)$, " " $(0,0,1$, $0)$, "“( $(0,1,0,0)$, "“ $(1,0,0,0)$, , and “( $0,0,0,0)$, respectively.
The second feature descriptor of NPS was the positionindependent k-mer frequency. We calculated the frequencies of all possible k-mer nucleotides in a site-centered nearby flanking window. However, the vector dimension increases rapidly with the increase of $k$ value, which leads to over-fitting. Thus, we set $k$ to 2, 3, and 4. Finally, the 41-length DNA sequence is transformed into a 500-dimensional vector. More details about this method are available in the Xiang et al. (2016).

\section{Pseudo Dinucleotide Composition (PseDNC)}

PseDNC combines local and global pattern information of sequences. We use a vector to represent the DNA sequence as given below,

$$
R=\left[\begin{array}{lllllll}
d_{1} & d_{2} & \cdots & d_{16} & d_{16+1} & \cdots & d_{16+\lambda}
\end{array}\right]^{T}
$$

where

$$
d_{u}=\left\{\begin{array}{cc}
\frac{f_{u}}{\sum_{i=1}^{16} f_{i}+w \sum_{j=1}^{\lambda} \theta_{j}} \quad(1 \leq u \leq 16) \\
\frac{w \theta_{u-16}}{\sum_{i=1}^{16} f_{i}+w \sum_{j=1}^{\lambda} \theta_{j}} \quad(16<u \leq 16+\lambda)
\end{array}\right.
$$


In the formulation above, $f_{u}(u=1,2, \cdots, 16)$ is the normalized occurrence frequency of the u-th non-overlapping dinucleotides in the sequence. The $w$ is the weight factor for balancing the component action of pseudo nucleotides. The $\theta_{j}$ is the $j$-th tier correlation factor that reflects the sequence order correlation between all the $j$-th most contiguous dinucleotides. What's more,

$$
\theta_{j}=\frac{1}{L-j-1} \sum_{i=1}^{L-j-1} C_{i, i+j} \quad(j=1,2, \cdots, \lambda ; \lambda<L)
$$

where

$$
C_{i, i+j}=\frac{1}{\mu} \sum_{g=1}^{\mu}\left[P_{g}\left(D_{i}\right)-P_{g}\left(D_{i+j}\right)\right]^{2}
$$

In the above two formulations, $L$ is the length of DNA sequence and the number $\lambda$ is an integer to reflect the correlation rank which is smaller than $L$. The $C_{i, i+j}$ is correlation function which is given above, where $P_{g}\left(D_{i}\right)$ is the numerical value of the $g$ th physicochemical property for the dinucleotide sequence $D_{i}$ in the DNA, and so as $P_{g}\left(D_{i+j}\right)$. The $\mu$ is the total number of correlation functions counted. It should be noticed that these values of physicochemical property were all subjected into a standard conversion by the formula below before substituting into the $P_{g}\left(D_{i}\right)$,

$$
P_{g}\left(D_{i}\right)=\frac{P_{g}^{0}\left(D_{i}\right)-\operatorname{ave}\left(P_{g}^{0}\left(D_{i}\right)\right)}{S D\left\{\operatorname{ave}\left(P_{g}^{0}\left(D_{i}\right)\right)\right\}}
$$

where the symbol ave() means getting the average of the values over the 16 different dinucleotides and $S D\{\}$ means the corresponding standard deviation. In the above equation, $P_{g}^{0}\left(D_{i}\right)$ is the original physicochemical property value for the dinucleotide. In this study, the following three physicochemical properties, namely enthalpy, entropy and free energy, are used to calculate the global or long-range sequence-order effects of the DNA. And their original values are given in Table S1 of Supplementary material.

Ultimately, using this feature descriptor, we obtained 22 features. More details about these formulas can be found in the references Chen et al. (2014, 2015a,b), Liu (2019), Liu et al. (2019b).

\section{Feature Fusion and Optimization Protocol}

Feature fusion has been successfully applied into bio-sequence analysis (Zhang et al., 2017; Tang et al., 2018; Wei et al., 2018a,b; Liu et al., 2019d) and other bioinformatics tasks (Liang et al., 2018; Zhang et al., 2018, 2019a,b; Gong et al., 2019; Wang et al., 2019). It refers to merge different types of feature representations to more comprehensively capture the characteristics of samples from different perspectives. In this study, to make better use of different information, we fused the following two feature representations. One is 500-dimensional feature vector via NPS and the other is 22-dimensional feature vector via PseDNC. Accordingly, we yielded 522-dimensional features.
Generally, the fused feature space probably contains irrelevant or mutual information, impacting the predictive performance. Therefore, feature optimization is a necessary step forwards capturing the most discriminative features from the original feature space, building the optimal predictive model. It can help to eliminate irrelevant or redundant features, so as to reduce feature dimension, improve model accuracy as well as reduce computational cost. On the other hand, selecting relevant features can simplify the model and make it easier to understand the process of data generation. So far, in order to solve these problems, various effective feature optimization methods have been proposed, such as analysis of variance (Feng et al., 2019), binomial distribution ( $\mathrm{Su}$ et al., 2018), minimal redundancy maximal relevance (Peng et al., 2005), and maximum relevance maximum distance (MRMD) (Zou et al., 2016; Chen W. et al., 2018).

To improve the feature representation ability, we used variance analysis in the filter method for feature selection. Its main idea is to calculate the variance of each feature by function f_classif in sklearn package. By doing so, we obtained the predictive contribution of each feature according to the corresponding f-value. The higher the f-value, the stronger the prediction ability. Afterwards, we selected the features one by one from high to low according to their fvalues, and trained the SVM model for each feature subset. Different feature subsets of different dimensions can produce different models, and thus different prediction results can be obtained. The feature subset with the highest accuracy is yielded as the optimal feature subset. The analysis of feature optimization results is discussed in section "RESULTS AND DISCUSSION".

\section{Support Vector Machine (SVM)}

SVM is a powerful machine learning method for classification, regression and other machine learning tasks. It has been successfully applied in various fields to deal with a series of supervised learning problems (Zhang et al., 2016; Bu et al., 2018; Liu and Li, 2019; Manavalan et al., 2019a,b). The main principle of SVM is to transform the import data into highdimensional feature space, and then determine the most suitable hyperplane for separating the samples in one class from another. After that, the trained hyperplane can be used to predict the unknown data. Based on this idea, a package namely LibSVM (Chih-chung and Chih-jen, 2011) was established to make the SVM more convenient to use. In this study, we implemented the SVM algorithm by using the LibSVM package. We chose the radial basis kernel $(\mathrm{RBF})$ as a learning function, and optimized the parameters like cost and gamma by grid search to determine the optimal classification hyperplane of SVM. Given a sequence sample, the SVM model can calculate its probability score to be true $6 \mathrm{~mA}$ sequence. If the probability is more than $50 \%$, it is considered to be the $6 \mathrm{~mA}$ sequence; otherwise, it is not the $6 \mathrm{~mA}$ sequence.

\section{Assessment of Predictive Ability}

There are three cross-validation methods namely independent dataset test, $\mathrm{n}$-fold cross-validation test and jackknife test 
TABLE 1 | Comparison of single feature and fused features.

\begin{tabular}{lccccc}
\hline Features & Sn (\%) & Sp (\%) & ACC (\%) & MCC & AUC \\
\hline NPS & 84.09 & 83.86 & 83.98 & 0.68 & 0.908 \\
PseDNC & 55.91 & 72.39 & 64.15 & 0.29 & 0.673 \\
Fused Features & 84.43 & 85.45 & 84.94 & 0.70 & 0.917
\end{tabular}

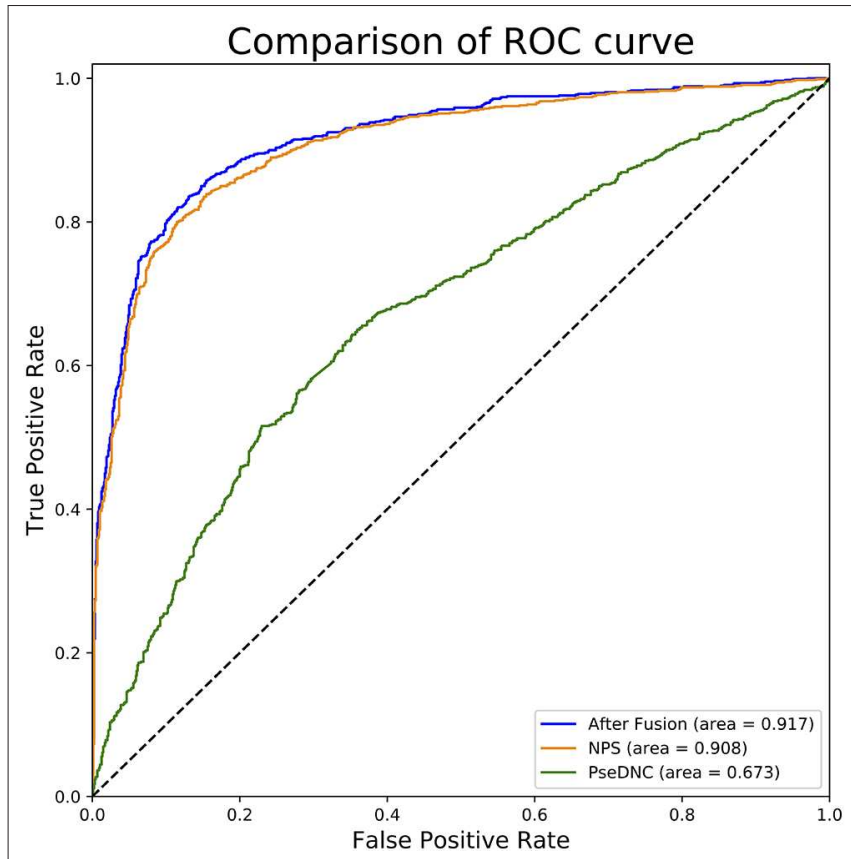

FIGURE 2 | ROC curves of single and fused features. This figure shows the $\mathrm{ROC}$ curve results of different features. The green curve characterizes ROC result of PseDNC and the orange one for NPS. The ROC curve of fused features is represented by blue.

in statistical prediction to evaluate expected success rate of predictors (Manavalan and Lee, 2017; Wei et al., 2017a,d; He et al., 2018; Manavalan et al., 2018; Liu and Zhu, 2019; Liu et al., 2019a). In this study, we used n-fold cross-validation to examine the quality of the model. In the $n$-fold cross-validation, the dataset was randomly divided into $n$ subsets, of which n1 subsets were used as training data and the remaining one as testing data. This process would be repeated $n$ times, each time using different testing data in turn. Corresponding accuracy and other evaluation metrics will be obtained in each test, and the average value of the evaluation index obtained from $n$-time results was used to evaluate the predictor. Generally, multiple nfold cross-validation (such as 10 times $\mathrm{n}$-fold cross-validation) is needed, and then its mean value is calculated to estimate the accuracy of the predictor.

Four metrics, sensitivity (Sn), specificity (Sp), accuracy (Acc) and Matthew's correlation coefficient (MCC), were used to evaluate the performance of the proposed method. The formulas

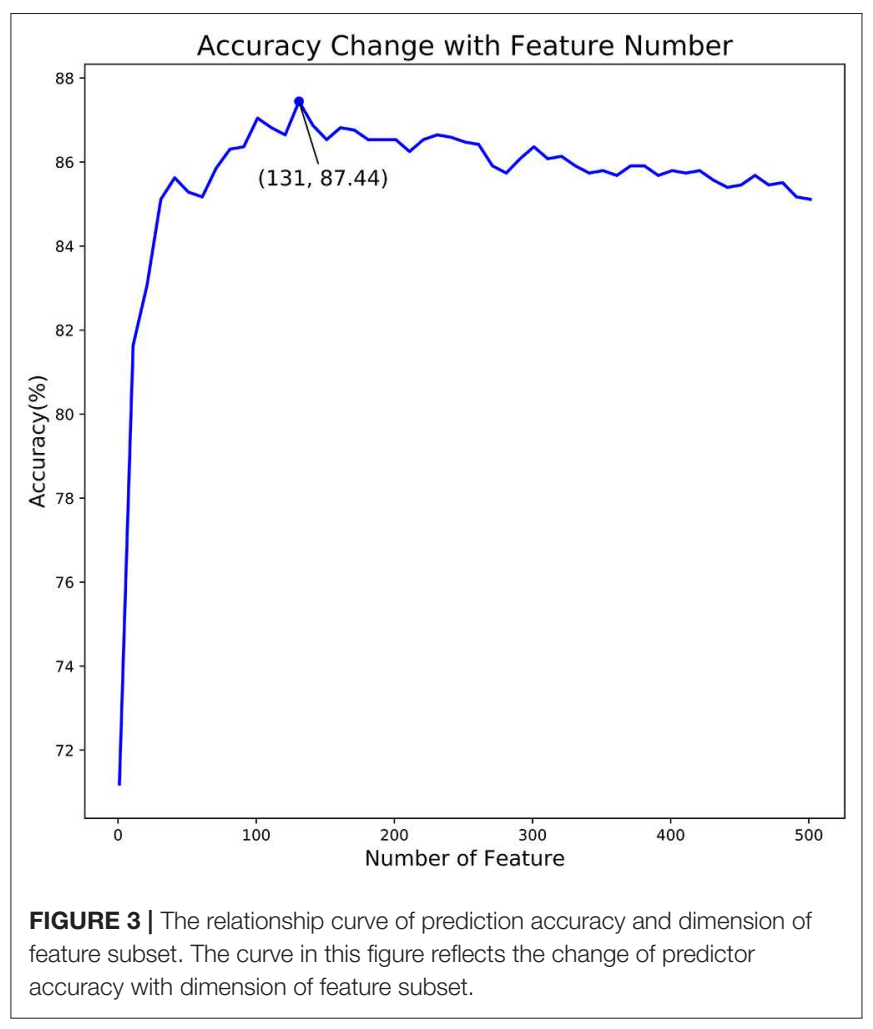

of these metrics are given below:

$$
\left\{\begin{array}{c}
S n=\frac{T P}{T P+F N} \quad 0 \leq S n \leq 1 \\
S p=\frac{T N}{T N+F P} \quad 0 \leq S p \leq 1 \\
A C C=\frac{T P+T N}{T P+F P+T N+F N} \quad 0 \leq A C C \leq 1 \\
M C C=\frac{T P \times T N-F P \times F N}{\sqrt{(T N+F N) \times(T N+F P) \times(T P+F N) \times(T P+F P)}} \\
-1 \leq M C C \leq 1
\end{array}\right.
$$

where, TP (True Positive) represents the number of positive samples correctly predicted; TN (True Negative) represents the number of negative samples correctly predicted; FP (False Positive) represents the number of negative samples incorrectly predicted to be the positives; FN (False Negative) represents the number of positive samples incorrectly predicted to be the negatives.

Moreover, we used the Receiver Operating Characteristic (ROC) curve to measure the overall performance of the predictive model. The area under the ROC curve (AUC) is to quantitively measure the quality of binary classifier. The closer the ROC curve is to the upper left corner, the better the performance of the predictor is. When the AUC value is closer to 0.5 , it means that this is a random predictor (Hanley and Mcneil, 1982).

\section{RESULTS AND DISCUSSION Comparison of Single and Fused Features} In this section, we investigated the impact of the feature fusion protocol on the predictive performance. We compared two 


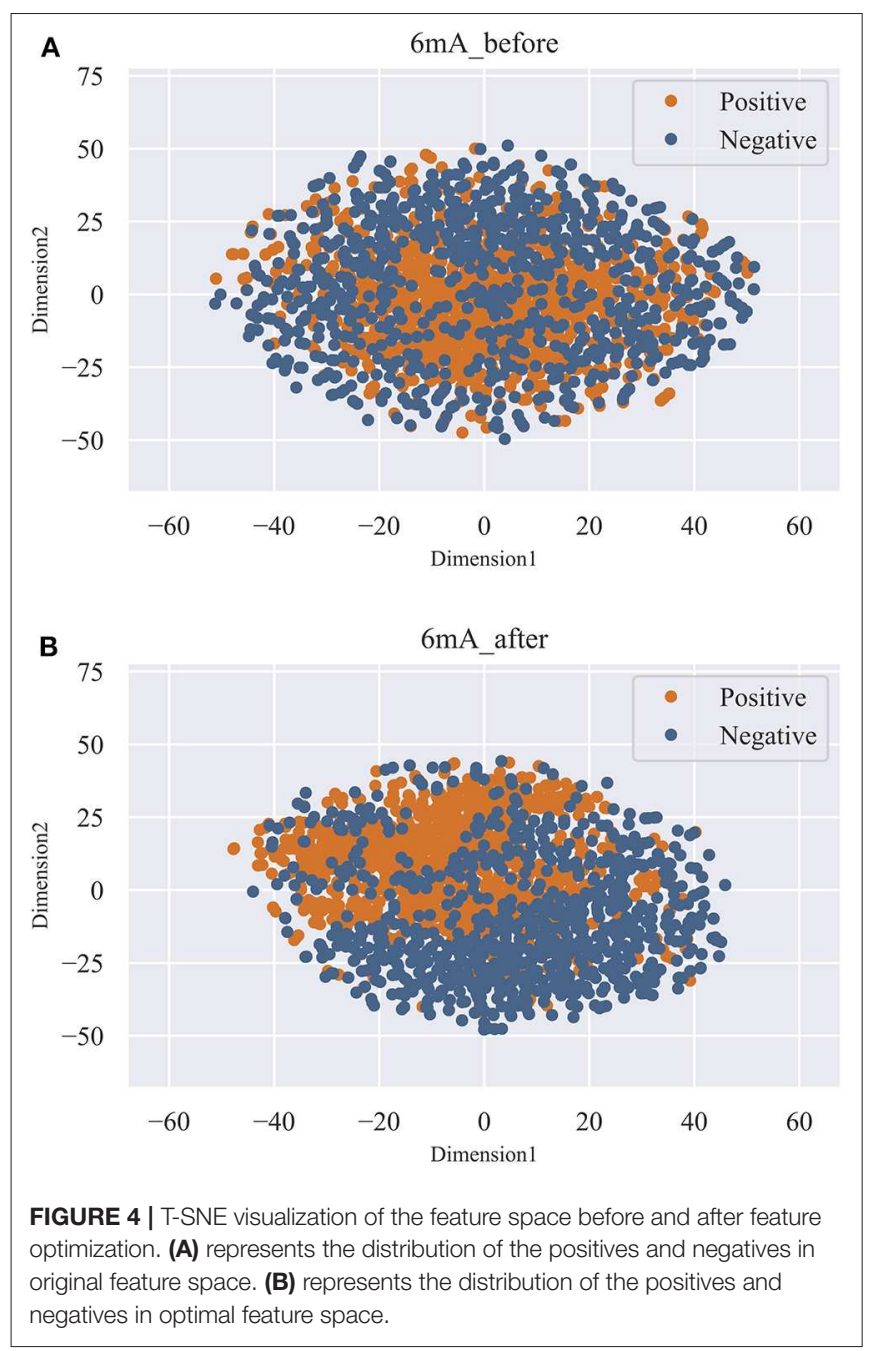

feature representations (NPS and PseDNC) with their fusion. They are evaluated with 10 -fold cross validation on the same benchmark dataset used in this study. The comparison results are presented in Table 1. It can be seen that the fused features improve the performances in all the metrics. To be specific, the Sn, Sp, ACC, MCC, and AUC is enhanced by $0.34,1.59,1,2$, and $0.9 \%$, as compared with the runner-up feature descriptor-NPS. For intuitive comparison, we further compared the ROC curves of different features in Figure 2. Similarly, the fused features show better performance than the single features. From the specific point of view in the Figure 2, the fused feature curve (the blue one) is closer to the upper left corner than the single feature curve. What's more, the AUC value of the fused feature is 0.917 , which is higher than that of the single feature. This figure and accurate data can more intuitively support the conclusion above. Together, the results suggest that the information in different features is complementary to better capture the characteristics specificity of $6 \mathrm{~mA}$ sites.

\section{Feature Optimization Results}

In the proposed feature optimization strategy, we firstly calculated the classification importance score of each feature in the feature set, and then the features are sorted from high to low according to their scores. Secondly, the feature in the sorted feature set is added to the feature subset one by one. Once a new feature is added to the feature subset, we obtained a new feature subset and train a new SVM model under its default parameters. We evaluated the performance of all feature subsets, respectively. The relationship between prediction accuracy and dimension of feature subset is illustrated in Figure 3.

As shown in Figure 3, we observed that the accuracy of the model increased rapidly as the feature number grows. Afterwards, the accuracy slightly declined as the feature number increases. When the feature number reached to 131, the model achieved the highest accuracy of $87.44 \%$. Thus, the 131 features are considered as the optimal and used to train our predictive model. Moreover, the feature optimization results evaluated with other evaluation metrics, like MCC and ROC, can be found in Table S2 of Supporting Information. To visually see how the feature space changes using feature optimization, we further compared the sample distribution between the original feature space and the optimal feature space, as depicted in Figure 4. It can be seen that the positives and negatives in the optimal feature space are more clearly distributed in two clear clusters as the original feature space. It demonstrates that using the feature optimization strategy, it helps to remove the irrelevant features and improve the feature representation ability.

\section{Comparison of Different Kernel Functions}

In this section, we compared the impact of RBF kernel function and other three kernel functions on the performance of our proposed model. They are Linear, Polynomial and Sigmoid. In this study, we used the same dataset to evaluate them. At the same time, they used the best feature subset after our fusion to show the performance. The 10-fold cross validation results can be found in Table S3 of Supplementary material. According to the results in Table S3, we can find easily that SVM model using RBF kernel function achieves the highest prediction accuracy of $87.44 \%$ and performs better in other prediction factors. Moreover, with the help of RBF kernel function, AUC of the model is also the highest among several other kernel functions. In general, these results show that RBF kernel function is superior to other kernel functions in this study.

\section{Comparison With Other Classifiers}

To measure the superiority of SVM, we selected several other classifiers to compare with SVM. There are Gradient Boosting Decision Tree (GBDT) (Liao et al., 2017), K-Nearest Neighbor (KNN), Logistic Regression (LR), Naive Bayes (NB), and Random Forest (RF) (Wei et al., 2017b,c; Lv et al., 2019; Ru et al., 2019). They are evaluated based on the same dataset used in this study with our fused feature set. The 10-flod cross validation results of prediction accuracy and AUC value are illustrated in Figure 5. In Figure 5A represents the comparison results of prediction accuracy of six classifiers, and Figure 5B represents the AUC value. As shown in Figure 5, we observed that the SVM got the highest score among the six classifiers not only in predictive accuracy but also AUC. The 10-fold cross validation results of other evaluation factors are illustrated in Table S4 of 

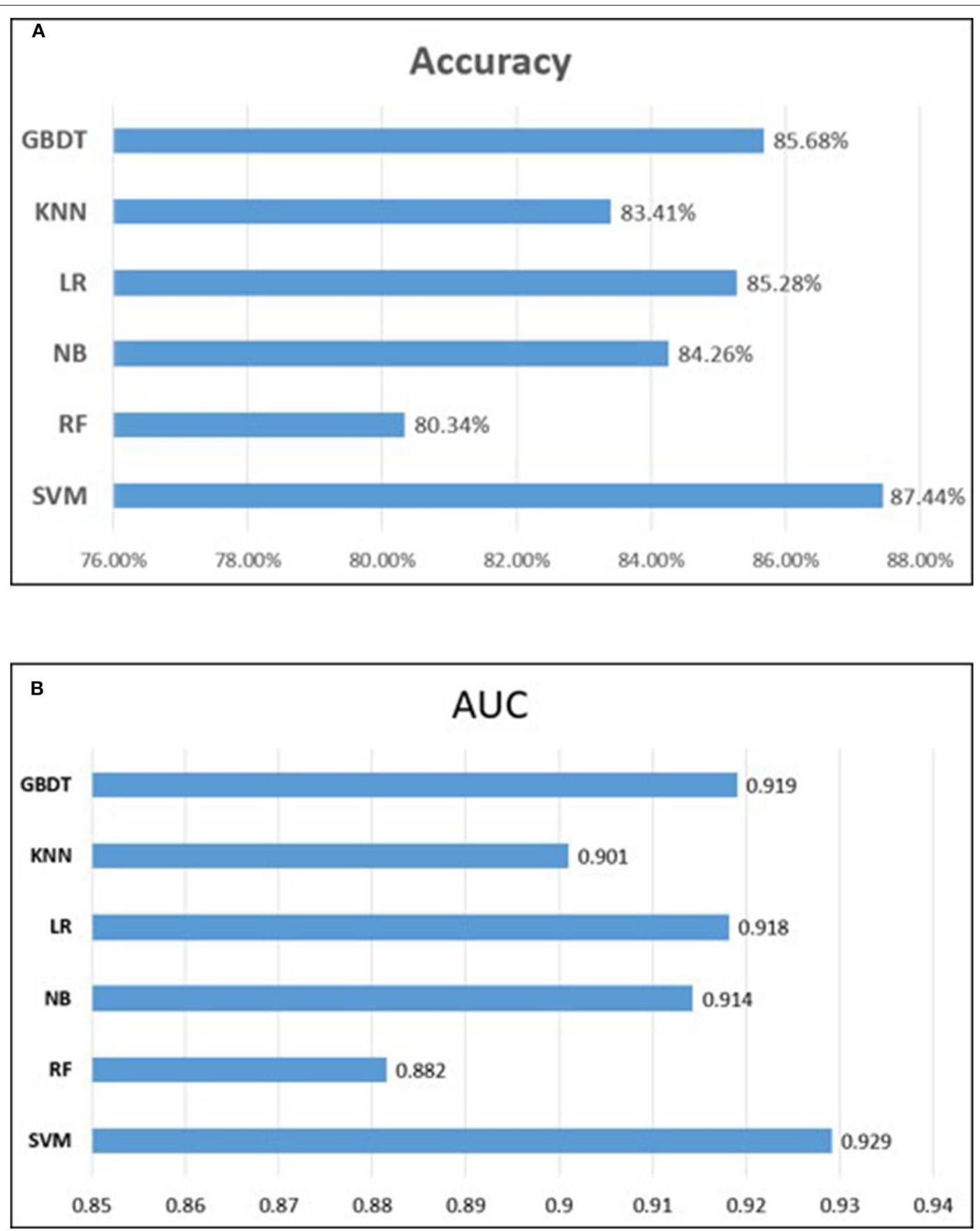

FIGURE 5 | Performance comparison of different classifiers. (A) represents the comparison results of prediction accuracy of six classifiers, and (B) represents the comparison results of auROC.

Supplementary material, which provide us with more specific classifier performance information. From Table S4, we can see that the SVM also performs better than other classifiers in other performance indicators. For intuitive comparison, we further compared their ROC curves as illustrated in Figure 6. As seen, SVM achieved 0.917 in terms of AUC, which is higher than GBDT and other classifiers. It can be seen from the figure that the ROC curve corresponding to SVM is at the top, which means that SVM has better classification performance than other classifiers. In general, these results demonstrate that SVM is better than other commonly used classifiers in this study.

\section{Comparison With Existing Predictors}

To measure the effectiveness of our predictive model$-6 \mathrm{mAPred}-\mathrm{FO}$, we compared the model with i6mA-Pred 


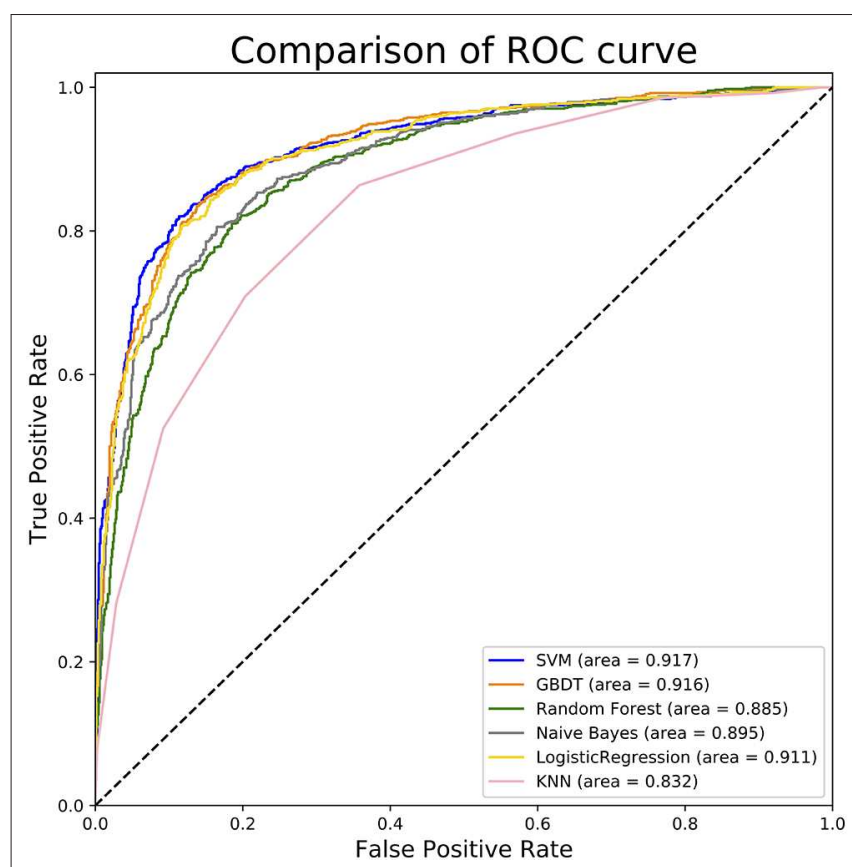

FIGURE 6 | ROC curves of different classifiers. This figure shows the ROC curve results of different classifiers. For example, according to legend, the blue curve characterizes ROC result of SVM and the orange one for GBDT. The value of auROC is also calculated after the name in the legend.

TABLE 2 | Comparison of the proposed 6mAPred-FO with existing predictors.

\begin{tabular}{lccccc}
\hline Method & Sn (\%) & Sp (\%) & ACC (\%) & MCC & AUC \\
\hline i6mA-Pred & 82.95 & 83.30 & 83.13 & 0.66 & 0.886 \\
iDNA6mA (5-step rule) & 86.70 & 86.59 & 86.64 & 0.732 & 0.931 \\
6mAPred-FO & 86.93 & 87.95 & 87.44 & 0.75 & 0.929
\end{tabular}

(Chen et al., 2019) and iDNA6mA (5-step rule) on the same dataset, which are the best two among existing predictors to identify the $6 \mathrm{~mA}$ site. The results are presented in Table 2. As shown in Table 2, i6mA-Pred obtains the accuracy of $83.13 \%$, sensitivity of $82.95 \%$, specificity of $83.30 \%$, MCC of 0.66 and AUC of 0.886 , while our prediction model obtains the accuracy of $87.44 \%$, sensitivity of $86.93 \%$, specificity of $87.95 \%$, MCC of 0.75 and AUC of 0.929 . Obviously, our method is superior to $16 \mathrm{~mA}$-Pred in all the metrics. Specifically, as compared to i6mA-Pred, our model achieved 4.31\%, 3.98\%, 4.65\%, 0.09 and 0.043 higher in terms of ACC, Sn, Sp, MCC, and AUC, respectively. This demonstrated that our feature representations are more effective to capture the characteristic specificity of $6 \mathrm{~mA}$ sites. In the Table 2, we also compared our predictor model with iDNA6mA (5-step rule). It can be seen that the accuracy of our $6 \mathrm{mAPred-FO}$ is $0.8 \%$ higher than iDNA $6 \mathrm{~mA}$ (5-step rule). All the other performance indicators except AUC value are slightly higher than those of iDNA6mA (5-step rule). Generally, it can be concluded that our 6mAPred-FO is better than existing predictors in distinguishing $6 \mathrm{~mA}$ sites from non-6mA sites.

\section{CONCLUSIONS}

In this study, we have proposed a new machine learning based $6 \mathrm{~mA}$ site predictor namely $6 \mathrm{mAPred}-\mathrm{FO}$. To sufficiently capture the characteristics of $6 \mathrm{~mA}$ sites, we have combined the information from two feature representations NPS and PseDNC, and further optimized the features by feature selection. Feature analysis results showed that as compared with the single feature descriptor, the fused features perform better, demonstrating that different information are complementary to improve the predictive performance. Moreover, feature selection is an effective strategy to optimize the feature space and improve the feature representation ability. We have also compared our $6 \mathrm{mAPred}-\mathrm{FO}$ with existing predictors on benchmark datasets. The comparative results showed that our approach improved the performance significantly in terms of multiple metrics like SN, SP, MCC, and AUC. This suggests that our feature fusion and selection scheme is more effective to represent $6 \mathrm{~mA}$ sites in comparison with existing features. From our study results, we can make a reasonable inference that the recognition of $6 \mathrm{~mA}$ site is closely related to the local and global pattern information represented by PseDNC. Then, the position specific information represented by NPS is fused to make our proposed algorithm more accurate for the recognition of $6 \mathrm{~mA}$ sites. In general, our method provides a more accurate model for biological scientists to identify $6 \mathrm{~mA}$ site in rice genome. In the future, we will pay more attention on deep learning (Liu et al., 2019c; Zou et al., 2019) for the accuracy improvement.

\section{DATA AVAILABILITY STATEMENT}

The datasets generated for this study can be found in the http:// server.malab.cn/6mAPred-FO/Download.html.

\section{AUTHOR CONTRIBUTIONS}

RC, YN, and DW: conceptualization. $\mathrm{XY}$ and RS: data curation. JC: writing-original draft preparation. GX and LW: writing-review and editing. JC: visualization. LW and GX: supervision.

\section{FUNDING}

This work is supported by National Natural Science Foundation of China (Grand Nos. 61701340, 61702431).

\section{SUPPLEMENTARY MATERIAL}

The Supplementary Material for this article can be found online at: https://www.frontiersin.org/articles/10.3389/fbioe. 2020.00502/full\#supplementary-material 


\section{REFERENCES}

Bu, H., Hao, J., Guan, J., and Zhou, S. (2018). Predicting enhancers from multiple cell lines and tissues across different developmental stages based on SVM method. Curr. Bioinformatics 13, 655-660. doi: $10.2174 / 1574893613666180726163429$

Campbell, J. L., and Kleckner, N. (1990). E. coli oriC and the dnaA gene promoter are sequestered from dam methyltransferase following the passage of the chromosomal replication fork. Cell 62, 967-979. doi: 10.1016/0092-8674(90)90271-F

Chen, H., Shu, H., Wang, L., Zhang, F., Li, X., Ochola, S. O., et al. (2018). Phytophthora methylomes are modulated by $6 \mathrm{~mA}$ methyltransferases and associated with adaptive genome regions. Genome Biol. 19:181. doi: 10.1186/s13059-018-1564-4

Chen, W., Feng, P., Ding, H., and Lin, H. (2018). Classifying included and excluded exons in exon skipping event using histone modifications. Front. Genet. 9:433. doi: 10.3389/fgene.2018.00433

Chen, W., Feng, P., Ding, H., Lin, H., and Chou, K. C. (2015a). iRNA-Methyl: Identifying $\mathrm{N}(6)$-methyladenosine sites using pseudo nucleotide composition. Anal. Biochem. 490, 26-33. doi: 10.1016/j.ab.2015.08.021

Chen, W., Lei, T. Y., Jin, D. C., Lin, H., and Chou, K. C. (2014). PseKNC: a flexible web server for generating pseudo K-tuple nucleotide composition. Anal. Biochem. 456, 53-60. doi: 10.1016/j.ab.2014.04.001

Chen, W., Lv, H., Nie, F., and Lin, H. (2019). i6mA-Pred: identifying DNA N6-methyladenine sites in the rice genome. Bioinformatics 35, 2796-2800. doi: 10.1093/bioinformatics/btz015

Chen, W., Yang, H., Feng, P., Ding, H., and Lin, H. (2017). iDNA4mC: identifying DNA N4-methylcytosine sites based on nucleotide chemical properties. Bioinformatics 33, 3518-3523. doi: 10.1093/bioinformatics/btx479

Chen, W., Zhang, X., Brooker, J., Lin, H., Zhang, L., and Chou, K. C. (2015b). PseKNC-General: a cross-platform package for generating various modes of pseudo nucleotide compositions. Bioinformatics 31, 119-120. doi: 10.1093/bioinformatics/btu602

Chih-chung, C., and Chih-jen, L. (2011). Libsvm: a library for support vector machines. ACM Trans. Intell. Syst. Technol. 2, 1-27. doi: 10.1145/1961189.1961199

Feng, C. Q., Zhang, Z. Y., Zhu, X. J., Lin, Y., Chen, W., Tang, H., et al. (2019). iTerm-PseKNC: a sequence-based tool for predicting bacterial transcriptional terminators. Bioinformatics 35, 1469-1477. doi: 10.1093/bioinformatics/bty827

Flusberg, B. A., Webster, D. R., Lee, J. H., Travers, K. J., Olivares, E. C., Clark, T. A., et al. (2010). Direct detection of DNA methylation during single-molecule, real-time sequencing. Nat. Methods 7, 461-465. doi: 10.1038/nmeth.1459

Fu, L., Niu, B., Zhu, Z., Wu, S., and Li, W. (2012). CD-HIT: accelerated for clustering the next-generation sequencing data. Bioinformatics 28, 3150-3152. doi: 10.1093/bioinformatics/bts565

Gong, Y., Niu, Y., Zhang, W., and Li, X. (2019). A network embedding-based multiple information integration method for the MiRNA-disease association prediction. BMC Bioinformatics 20:468. doi: 10.1186/s12859-019-3063-3

Greer, E. L., Blanco, M. A., Gu, L., Sendinc, E., Liu, J., Aristizabal-Corrales, D., et al. (2015). DNA Methylation on N6-Adenine in C. elegans. Cell 161, 868-878. doi: 10.1016/j.cell.2015.04.005

Hanley, J. A., and Mcneil, B. J. (1982). The meaning and use of the area under a receiver operating characteristic (ROC) curve. Radiology 143, 29-36. doi: 10.1148/radiology.143.1.7063747

He, W., Jia, C., Duan, Y., and Zou, Q. (2018). 70ProPred: a predictor for discovering sigma70 promoters based on combining multiple features. $B M C$ Syst. Biol. 12(Suppl. 4):44. doi: 10.1186/s12918-018-0570-1

Krais, A. M., Cornelius, M. G., and Schmeiser, H. H. (2010). Genomic $\mathrm{N}(6)$-methyladenine determination by MEKC with LIF. Electrophoresis 31, 3548-3551. doi: 10.1002/elps.201000357

Li, B., Du, K., Gu, S., Xie, J., Liang, T., Xu, Z., et al. (2019). Epigenetic DNA modification N(6)-Methyladenine inhibits DNA replication by DNA polymerase of pseudomonas aeruginosa Phage PaP1. Chem. Res. Toxicol. 32, 840-849. doi: 10.1021/acs.chemrestox.8b00348

Liang, D., Wang, H., Song, W., Xiong, X., Zhang, X., Hu, Z., et al. (2016). The decreased N(6)-methyladenine DNA modification in cancer cells. Biochem. Biophys. Res. Commun. 480, 120-125. doi: 10.1016/j.bbrc.2016.09.136
Liang, S., Ma, A., Yang, S., Wang, Y., and Ma, Q. (2018). A review of matched-pairs feature selection methods for gene expression data analysis. Comput. Struct. Biotechnol. J. 16, 88-97. doi: 10.1016/j.csbj.2018.02.005

Liao, Z., Li, D., Wang, X., Li, L., and Zou, Q. (2016). Cancer diagnosis through isomiR expression with machine learning method. Curr. Bioinform. 13, 57-63. doi: 10.2174/1574893611666160609081155

Liao, Z., Wan, S., He, Y., and Zou, Q. (2017). Classification of small GTPases with hybrid protein features and advanced machine learning techniques. Curr. Bioinform. 12, 1-9. doi: 10.2174/1574893612666171121162552

Linn, S., and Arber, W. (1968). Host specificity of DNA produced by Escherichia coli, X. In vitro restriction of phage fd replicative form. Proc. Natl. Acad. Sci. U.S.A. 59, 1300-1306. doi: 10.1073/pnas.59.4.1300

Liu, B. (2019). BioSeq-Analysis: a platform for DNA, RNA and protein sequence analysis based on machine learning approaches. Brief Bioinform. 20, 1280-1294. doi: 10.1093/bib/bbx165

Liu, B., Chen, S., Yan, K., and Weng, F. (2019a). iRO-PsekGCC: Identify DNA Replication Origins Based on Pseudo k-Tuple GC Composition. Front. Genet 10:842. doi: 10.3389/fgene.2019.00842

Liu, B., Gao, X., and Zhang, H. (2019b). BioSeq-Analysis2.0: an updated platform for analyzing DNA, RNA and protein sequences at sequence level and residue level based on machine learning approaches. Nucleic Acids Res. 47:e127. doi: 10.1093/nar/gkz740

Liu, B., Li, C. C., and Yan, K. (2019c). DeepSVM-fold: protein fold recognition by combining support vector machines and pairwise sequence similarity scores generated by deep learning networks. Brief Bioinform. doi: 10.1093/bib/bbz098

Liu, B., and Li, K. (2019). iPromoter-2L2.0: Identifying promoters and their types by combining smoothing cutting window algorithm and sequence-based features. Mol. Ther. Nucleic Acids 18, 80-87. doi: 10.1016/j.omtn.2019.08.008

Liu, B., and Zhu, Y. (2019). ProtDec-LTR3.0: protein remote homology detection by incorporating profile-based features into learning to rank. IEEE Access 7, 102499-102507. doi: 10.1109/ACCESS.2019.2929363

Liu, S., Zhao, X., Zhang, G., Li, W., Liu, F., Liu, S., et al. (2019d). PredLncGFStack: a global sequence feature based on a stacked ensemble learning method for predicting lncRNAs from transcripts. Genes (Basel) 10:672. doi: 10.3390/genes10090672

Luria, S. E., and Human, M. L. (1952). A nonhereditary, host-induced variation of bacterial viruses. J. Bacteriol 64, 557-569. doi: 10.1128/JB.64.4.557-5 69.1952

Lv, Z., Jin, S., Ding, H., and Zou, Q. (2019). A random forest sub-golgi protein classifier optimized via dipeptide and amino acid composition features. Front. Bioeng. Biotechnol. 7:215. doi: 10.3389/fbioe.2019.00215

Manavalan, B., Basith, S., Shin, T. H., Wei, L., and Lee, G. (2019a). mAHTPred: a sequence-based meta-predictor for improving the prediction of antihypertensive peptides using effective feature representation. Bioinformatics 35 , 2757-2765. doi: 10.1093/bioinformatics/bty1047

Manavalan, B., Basith, S., Shin, T. H., Wei, L., and Lee, G. (2019b). Meta-4mCpred: a sequence-based meta-predictor for accurate DNA $4 \mathrm{mC}$ site prediction using effective feature representation. Mol. Ther. Nucleic Acids 16, 733-744. doi: 10.1016/j.omtn.2019.04.019

Manavalan, B., and Lee, J. (2017). SVMQA: support-vector-machine-based protein single-model quality assessment. Bioinformatics 33, 2496-2503. doi: 10.1093/bioinformatics/btx222

Manavalan, B., Shin, T. H., and Lee, G. (2018). PVP-SVM: sequence-based prediction of phage virion proteins using a support vector machine. Front. Microbiol. 9:476. doi: 10.3389/fmicb.2018.00476

Meselson, M., and Yuan, R. (1968). DNA restriction enzyme from E. coli. Nature 217, 1110-1114. doi: 10.1038/2171110a0

O’Brown, Z. K., and Greer, E. L. (2016). N6-Methyladenine: a conserved and dynamic DNA mark. Adv. Exp. Med. Biol. 945, 213-246. doi: 10.1007/978-3-319-43624-1_10

Peng, H., Long, F., and Ding, C. (2005). Feature selection based on mutual information: criteria of max-dependency, max-relevance, and minredundancy. IEEE Trans. Pattern Anal. Mach. Intell. 27, 1226-1238. doi: 10.1109/TPAMI.2005.159

Pomraning, K. R., Smith, K. M., and Freitag, M. (2009). Genome-wide high throughput analysis of DNA methylation in eukaryotes. Methods 47, 142-150. doi: 10.1016/j.ymeth.2008.09.022 
Pukkila, P. J., Peterson, J., Herman, G., Modrich, P., and Meselson, M. (1983). Effects of high levels of DNA adenine methylation on methyl-directed mismatch repair in Escherichia coli. Genetics 104, 571-582.

Robbins-Manke, J. L., Zdraveski, Z. Z., Marinus, M., and Essigmann, J. M. (2005). Analysis of global gene expression and double-strand-break formation in DNA adenine methyltransferase- and mismatch repair-deficient Escherichia coli. J. Bacteriol. 187, 7027-7037. doi: 10.1128/JB.187.20.7027-7037.2005

Ru, X., Li, L., and Zou, Q. (2019). Incorporating distance-based top-n-gram and random forest to identify electron transport proteins. J. Proteome Res. 18, 2931-2939. doi: 10.1021/acs.jproteome.9b00250

Su, Z. D., Huang, Y., Zhang, Z. Y., Zhao, Y. W., Wang, D., Chen, W., et al. (2018). iLoc-lncRNA: predict the subcellular location of lncRNAs by incorporating octamer composition into general PseKNC. Bioinformatics 34, 4196-4204. doi: 10.1093/bioinformatics/bty508

Tahir, M., Tayara, H., and Chong, K. T. (2019). iDNA6mA (5-step rule): identification of DNA N6-methyladenine sites in the rice genome by intelligent computational model via Chou's 5-step rule. Chemometr. Intell. Lab. Systems 189, 96-101. doi: 10.1016/j.chemolab.2019.04.007

Tang, G., Shi, J., Wu, W., Yue, X., and Zhang, W. (2018). Sequence-based bacterial small RNAs prediction using ensemble learning strategies. BMC Bioinformatics 19, 13-23. doi: 10.1186/s12859-018-2535-1

Wang, Y., Yang, S., Zhao, J., Du, W., Liang, Y., Wang, C., et al. (2019). Using machine learning to measure relatedness between genes: a multi-features model. Sci. Rep. 9, 1-15. doi: 10.1038/s41598-019-40780-7

Wei, L., Chen, H., and Su, R. (2018a). M6APred-EL: a sequence-based predictor for identifying N6-methyladenosine sites using ensemble learning. Mol. Ther. Nucleic Acids 12, 635-644. doi: 10.1016/j.omtn.2018.07.004

Wei, L., Wan, S., Guo, J., and Wong, K. K. (2017a). A novel hierarchical selective ensemble classifier with bioinformatics application. Artif. Intell. Med. 83, 82-90. doi: 10.1016/j.artmed.2017.02.005

Wei, L., Xing, P., Su, R., Shi, G., Ma, Z. S., and Zou, Q. (2017b). CPPred-RF: a sequence-based predictor for identifying cell-penetrating peptides and their uptake efficiency. J. Proteome Res. 16, 2044-2053. doi: 10.1021/acs.jproteome.7b00019

Wei, L., Xing, P., Tang, J., and Zou, Q. (2017c). PhosPred-RF: a novel sequencebased predictor for phosphorylation sites using sequential information only. IEEE Trans. Nanobiosc. 16, 240-247. doi: 10.1109/TNB.2017.2661756

Wei, L., Xing, P., Zeng, J., Chen, J., Su, R., and Guo, F. (2017d). Improved prediction of protein-protein interactions using novel negative samples, features, and an ensemble classifier. Artif. Intell. Med. 83, 67-74. doi: 10.1016/j.artmed.2017.03.001

Wei, L., Zhou, C., Chen, H., Song, J., and Su, R. (2018b). ACPredFL: a sequence-based predictor using effective feature representation to improve the prediction of anti-cancer peptides. Bioinformatics 34, 4007-4016. doi: 10.1093/bioinformatics/bty451

Xiang, S., Yan, Z., Liu, K., Zhang, Y., and Sun, Z. (2016). AthMethPre: a web server for the prediction and query of mRNA m(6)A sites in Arabidopsis thaliana. Mol. Biosyst. 12, 3333-3337. doi: 10.1039/C6MB00536E
Ye, P., Luan, Y., Chen, K., Liu, Y., Xiao, C., and Xie, Z. (2017). MethSMRT: an integrative database for DNA N6-methyladenine and N4-methylcytosine generated by single-molecular real-time sequencing. Nucleic Acids Res. 45, D85-D89. doi: 10.1093/nar/gkw950

Zhang, N., Sa, Y., Guo, Y., Lin, W., Wang, P., and Feng, Y. (2016). Discriminating ramos and jurkat cells with image textures from diffraction imaging flow cytometry based on a support vector machine. Curr. Bioinform. 11, 50-56. doi: 10.2174/1574893611666160608102537

Zhang, W., Jing, K., Huang, F., Chen, Y., Li, B., Li, J., et al. (2019a). SFLLN: a sparse feature learning ensemble method with linear neighborhood regularization for predicting drug-drug interactions. Inf. Sci. 497, 189-201. doi: 10.1016/j.ins.2019.05.017

Zhang, W., Li, Z., Guo, W., Yang, W., and Huang, F. (2019b). A fast linear neighborhood similarity-based network link inference method to predict microRNA-disease associations. IEEE/ACM Trans. Comput. Biol. Bioinform. doi: 10.1109/TCBB.2019.2931546

Zhang, W., Yue, X., Tang, G., Wu, W., Huang, F., and Zhang, X. (2018). SFPEL-LPI: Sequence-based feature projection ensemble learning for predicting LncRNA-protein interactions. PLoS Comput. Biol. 14:e1006616. doi: 10.1371/journal.pcbi.1006616

Zhang, W., Zhu, X., Fu, Y., Tsuji, J., and Weng, Z. (2017). Predicting human splicing branchpoints by combining sequence-derived features and multi-label learning methods. BMC Bioinformatics 18(Suppl. 13):464. doi: 10.1186/s12859-017-1875-6

Zhou, C., Wang, C., Liu, H., Zhou, Q., Liu, Q., Guo, Y., et al. (2018). Identification and analysis of adenine $\mathrm{N}(6)$-methylation sites in the rice genome. Nat Plants 4, 554-563. doi: 10.1038/s41477-0180214-X

Zou, Q., Xing, P., Wei, L., and Liu, B. (2019). Gene2vec: gene subsequence embedding for prediction of mammalian $\mathrm{N}$ (6)-methyladenosine sites from mRNA. RNA 25, 205-218. doi: 10.1261/rna.0691 12.118

Zou, Q., Zeng, J., Cao, L., and Ji, R. (2016). A novel features ranking metric with application to scalable visual and bioinformatics data classification. Neurocomputing 173, 346-354. doi: 10.1016/j.neucom.2014. 12.123

Conflict of Interest: The authors declare that the research was conducted in the absence of any commercial or financial relationships that could be construed as a potential conflict of interest.

Copyright (c) 2020 Cai, Wang, Chen, Niu, Ye, Su, Xiao and Wei. This is an openaccess article distributed under the terms of the Creative Commons Attribution License (CC BY). The use, distribution or reproduction in other forums is permitted, provided the original author(s) and the copyright owner(s) are credited and that the original publication in this journal is cited, in accordance with accepted academic practice. No use, distribution or reproduction is permitted which does not comply with these terms. 